\title{
DES SURFACES FONCTIONNALISÉES inspirées par la nature
}

\author{
Marc FAUCON \\ Laura GEMINI \\ Alphanov
}

marc.faucon@alphanov.com laura.gemini@alphanov.com

Au cours de la dernière décennie, les processus laser sont devenus le choix préféré pour de nombreuses industries. Les lasers permettent en effet d'adapter chaque processus à une large gamme de matériaux par la simple variation des différents paramètres du procédé, tels que la longueur d'onde, la durée, l'énergie et la fréquence des impulsions. Dans ce contexte, les lasers à impulsions ultra-courtes (avec une durée de l'ordre de quelques dizaines de femtosecondes) permettent, entre autres, de fonctionnaliser les surfaces des matériaux en adaptant leurs propriétés à des besoins spécifiques.

\section{Texturation par laser ultra-court: du nanomètre au micromètre}

Différents types de nano et microstructures peuvent être générés par texturation laser (figure 1). En règle générale, à faibles doses d'éclairement laser (i.e. énergie totale déposée par unité de surface texturée), il est possible d'observer la présence d'ondulations, également appelées ripples ou LIPSS (figure 1a). Si le laser a une polarisation linéaire, ce type de nanostructure est composé de lignes périodiques avec une direction perpendiculaire à la polarisation du laser et un espacement de l'ordre de la longueur d'onde du laser. Lorsque la dose augmente, les ondulations se transforment en microstructures périodiques avec une direction parallèle à la polarisation du laser (figure 1b). Enfin, lorsque la dose augmente encore, les effets thermiques prennent un rôle prépondérant dans l'évolution de la morphologie de surface, et des microstructures coniques, également appelées spikes (figure 1c), sont générées uniformément sur la surface. La taille de ces structures augmente avec la dose jusqu'à saturation à environ plusieurs dizaines de microns. l'échelle du micron, voire l'échelle nanométrique). Pour la mise en œuvre de ces procédés au sein de l'industrie, des lasers plus compacts, plus robustes et offrant des vitesses d'usinage plus importantes, donc une puissance moyenne plus élevée, ont été développés. Aujourd'hui, les sources lasers femtoseconde peuvent atteindre des dizaines de watts avec des fréquences de plusieurs centaines de $\mathrm{kHz}$. Le couplage avec un scanner ayant une vitesse de balayage de plusieurs dizaines de mètres par seconde permet d'atteindre des vitesses d'usinage de l'ordre de quelques secondes par centimètre carré $[1,2]$.

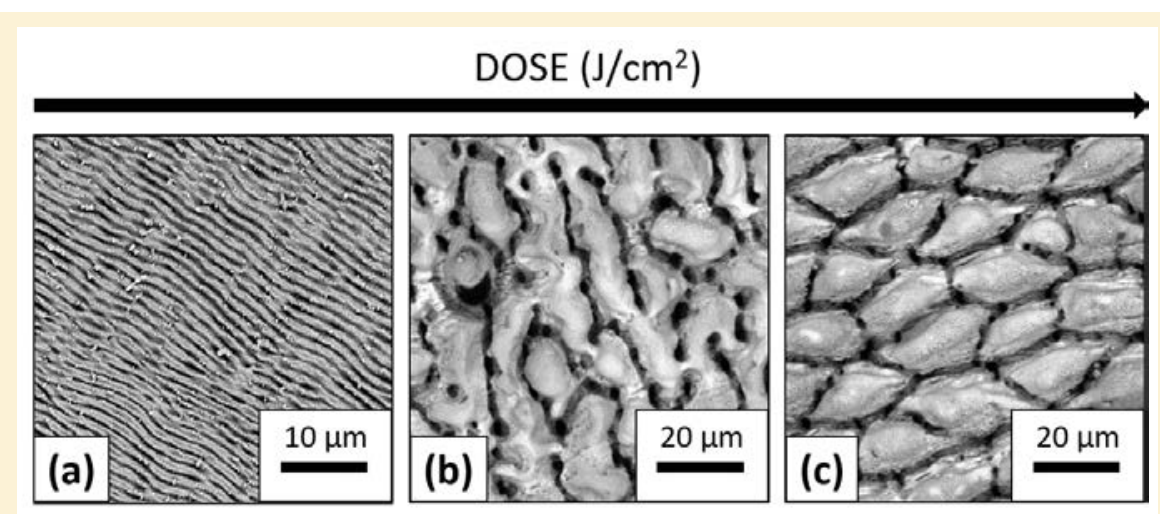

Figure 1. Différents types de nano et microstructures générées avec une dose faible (a, LIPSS), intermédiaire (b, microstructures périodiques), et élevée (c, spikes) d'éclairement laser. 

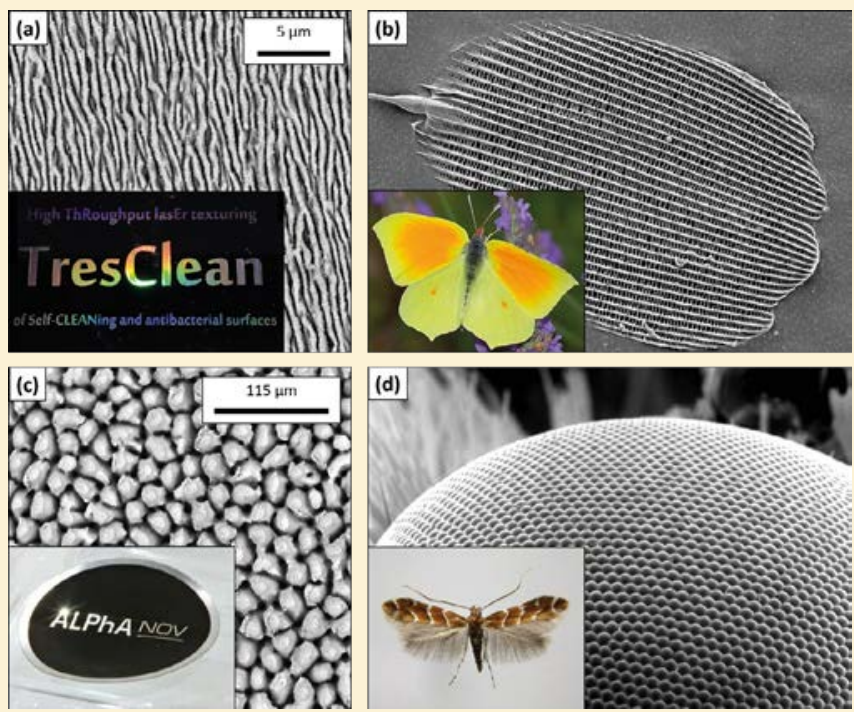

Figure 2. (b) Effet holographique sur les ailes de papillon (Cleopatra Gonepteryx) et (d) le noir de l'œil de mite (Lepidoptera) reproduit par texturation laser $(\mathrm{a}, \mathrm{c})$.

L'évolution de la morphologie de surface de LIPSS à microstructures coniques peut être expliquée par des phénomènes d'interférences pour les LIPSS et par les déplacements de la matière dus à des effets thermiques dans le cas des spikes. L' origine des LIPSS est généralement attribuée à une interférence entre le faisceau laser incident et des ondes de surface électromagnétiques qui se propagent sur la surface à la suite de l'interaction intensive avec le laser [3]. Dans un régime de valeurs élevées de dose, les effets thermiques l'emportent sur ceux de l'interférence et le matériau se réarrange par mouvements thermiques pour former les microstructures coniques [4].

Chaque type de nano ou microstructure donne à la surface du matériau des propriétés spécifiques. Dans le cas des LIPSS, la lumière ambiante incidente est diffractée sur la surface par la grille des ondulations et produit un effet visuel holographique (figure 2a), comme il est possible d' observer dans le cas d'ailes de papillon (figure 2b). Dans le cas des spikes, la surface acquiert d'autres propriétés optiques: la surface paraît noire et peut atteindre des valeurs très faibles de réflectivité, jusqu'à $5 \%$, en absorbant presque totalement la lumière incidente (figure $2 c$ ). Cet effet est similaire à ce qui se trouve dans les yeux de certains insectes nocturnes: la présence de spikes empêchant la réflexion entre l'air et la cornée est à l'origine de la qualité de leur vision de nuit (figure $2 d$ ).

\section{Texturation pour les processus industriels}

La texturation par laser présente, à l'instar des autres procédés laser, des qualités intrinsèques particulièrement prisées du monde industriel. Procédé sans contact, elle peut ainsi être réalisée à distance et sur des pièces de formes très variées, voire sur des surfaces difficiles d'accès pour des outils traditionnels. C'est par ailleurs un procédé en une seule étape, qui ne nécessite donc ni pré-, ni post-traitement et qui ne met en cuvre aucun produit chimique.

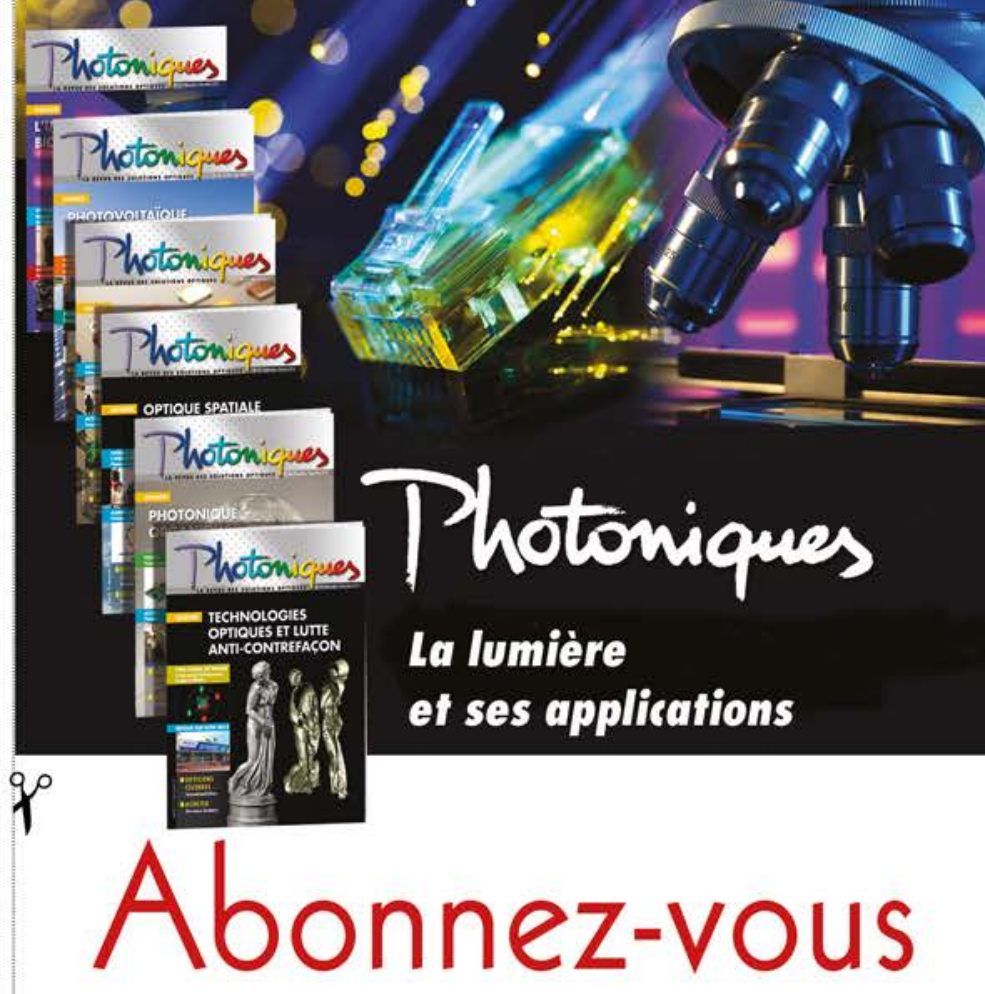

\begin{tabular}{|c|c|}
\hline \multicolumn{2}{|c|}{ abonnement ( 1 an / 5 numéros + hors série) } \\
\hline institutions & $\begin{array}{c}\text { étudiants } \\
\text { [à titre individuel, sur justificatiff) }\end{array}$ \\
\hline \multicolumn{2}{|c|}{ France/UE } \\
\hline papier + électronique : $98 € \pi c$ & papier + électronique : $62 € \pi c$ \\
\hline électronique : $71,47 € \pi c$ & électronique : $45,95 € \pi c$ \\
\hline \multicolumn{2}{|c|}{ Reste du monde } \\
\hline papier + électronique : $128 €$ & papier + électronique : $90 €$ \\
\hline électronique : $70 €$ & électronique : $45 €$ \\
\hline
\end{tabular}

Nom

Prénom

Société

Adresse

Code postal $\sqcup \amalg \sqcup \sqcup$ Ville

Pays

Téléphone

E-mail .

$\square$ Je règle par carte bancaire :

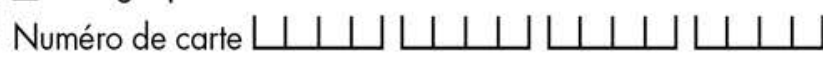

Date de fin de validité $\bigsqcup \sqcup \sqcup \sqcup$ Cryptogramme visuel $\bigsqcup \sqcup \sqcup$ (code inscrit au dos de ma carte)

$\square$ Je joins un chèque de............... euros à l'ordre d'EDP Sciences

Merci de me faire parvenir une facture acquittée

Signature :

Bulletin à retourner à

\section{edpsciences}

EDP Sciences, 17 avenue du Hoggar,

P.A. de Courtabœuf, B.P. 112, 91944 Les Ulis Cedex A

ou par mail : abonnements@edpsciences.org

Abonnez-vous en ligne : www.photoniques.com 

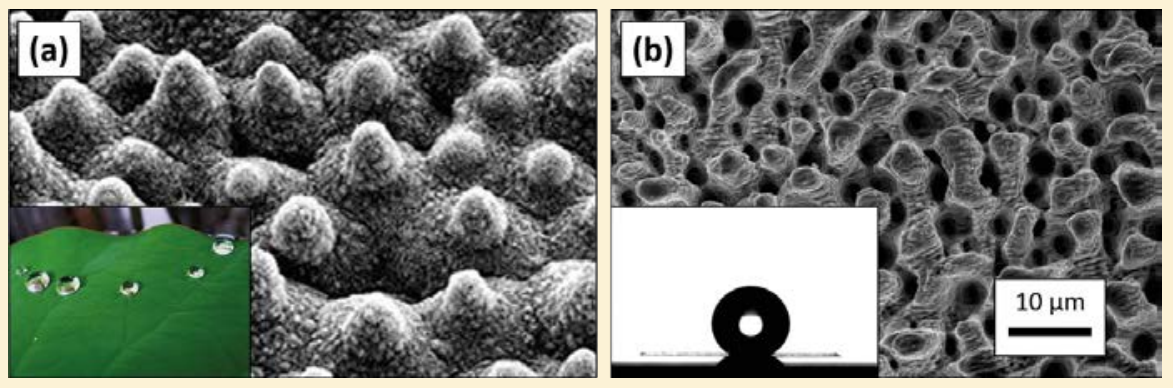

Figure 3. Hydrophobie observée pour les feuilles de lotus (a ) et reproduction par texturation laser de cet effet $(b)$.

Dans ce contexte, les structures nanométriques et micrométriques trouvent des applications différentes. Les colorations apportées par les ondulations des LIPSS trouvent des applications naturelles dans tous les secteurs sensibles aux aspects décoratifs (joaillerie, parfumerie, horlogerie) mais sont aussi utilisées pour des marquages anti-contrefaçon. Les microstructures coniques sont plutôt utilisées dans des applications basées sur la capture de la lumière, telles que les détecteurs infrarouge et les panneaux photovoltaïques pour augmenter leur taux de conversion de photons incidents.

Une propriété commune à ces structures est le changement de la mouillabilité de la surface, comme il est observé pour la feuille de lotus (figure 3a). Dans ce contexte, ALPhANOV est maintenant partenaire du projet européen TresClean (www.tresclean.eu). Le but principal de ce projet est de produire par texturation laser à haute productivité des surfaces antibactériennes et hydrophobes pour une utilisation dans l'industrie alimentaire et les appareils ménagers. De cette manière, il est possible de réduire la maintenance et allonger la durée de vie des outils traités. Le caractère hydrophobe de la surface est généralement attribué à la présence d'air entre la surface texturée et le liquide, qui réduit les points de contact, facilitant le roulement des gouttes de liquide. Aujourd'hui, faces super-hydrophobes présentant ALPhANOV a réussi à obtenir des sur-

\section{POUR EN SAVOIR PLUS} over large area, Applied Surface Science, 386, 65 (2016) un angle de contact entre la goutte de liquide et la surface supérieure à $150^{\circ}$, avec une vitesse d'usinage de quelques secondes par centimètre carre (figure 3b) [5].

Ces résultats du projet TresClean débuté en avril 2016 montrent d'ores et déjà la capacité à conserver la génération de structures à morphologie complexe à haute cadence de tir et haute puissance moyenne jusqu'à $100 \mathrm{~W}$. Ces résultats prometteurs permettent de franchir un nouveau palier pour la fonctionnalisation de grandes surfaces soit en inscription directe, soit pour la gravure de moules d'injection. Les enjeux futurs seront de conserver ces morphologies avec des puissances moyennes toujours plus élevées malgré une accumulation thermique importante en raison du faible temps entre chaque impulsion (de l'ordre de $0,1 \mu \mathrm{s})$. Le projet TresClean répondra à ces nouveaux enjeux avec une fin du projet programmée pour octobre 2019.

[1] Faucon, M., Laffitte, A., Lopez, J., Kling, R., Surface blackening by laser texturing with high repetition rate femtosecond laser up to $1 \mathrm{MHz}$, Proceedings of SPIE, Vol. 8972 (2014)

[2] Mincuzzi, G., Gemini, L., Faucon, M., Kling, R., Extending ultra-short pulse laser texturing

[3] Bonse, J., Höhm, S., Kirner, S.V., Rosenfeld, A., Krüger, J., Laser-induced periodic surface structures - a scientific evergreen, IEEE Journal of Selected Topics in Quantum Electronics, 23, 3 (2017)

[4] Tsibidis, G.D., Fotakis, C., Stratakis, E., Physical Review B 92, 041405 (2015)

[5] Gemini, L., Faucon, M., Romoli, L., Kling, R., High throughput laser texturing of super-hydrophobic surfaces on steel, Proceedings of SPIE 10092, 100921G (2017) 